\section{BMJ Open Respiratory Research}

\title{
Reducing hospital admissions for COPD: perspectives following the Christchurch Earthquake
}

Michael Epton, ${ }^{1}$ Carol Limber, ${ }^{2}$ Carolyn Gullery, ${ }^{2}$ Graham McGeoch, ${ }^{3}$ Brett Shand, ${ }^{3}$ Rose Laing, ${ }^{4}$ Simon Brokenshire, ${ }^{5}$ Andrew Meads, ${ }^{5}$ Rachel Nicholson-Hitt ${ }^{5}$

\section{ABSTRACT}

The devastating 2011 earthquake in Christchurch destroyed or badly damaged healthcare infrastructure, including Christchurch Hospital. This forced change in management of exacerbations of chronic obstructive pulmonary disease (COPD), which until that point had frequently led to admission to hospital and focused attention on providing safe community options for care. This paper describes the process of understanding factors contributing to high admission frequency with exacerbations of COPD and also describes a process of change, predominantly to healthcare delivery systems and philosophies, and the subsequent outcomes. What became clear in understanding admissions with COPD to Christchurch Hospital was that the behaviour of the patient, in the context of exacerbations, and the subsequent response of the system to the patient, led to admission being the default option, in spite of low severity of the exacerbation itself. By altering systems' responses to exacerbations, with a linked care process between ambulances, community care and hospitals, we were able to safely reduce admissions for COPD, with a sustained overall reduction in bed-day occupancy for COPD of $\sim 48 \%$. We would encourage these discussions and changes to occur without the stimulus of an earthquake in your healthcare environment! (s) 2018. Re-use permitted under CC BY-NC. No commercial re-use. See rights and permissions. Published by BMJ

${ }^{1}$ Department of Respiratory Medicine, Christchurch Hospital, Christchurch, New Zealand

${ }^{2}$ Planning and Funding and Decision Support, Canterbury District Health Board, Christchurch, New Zealand ${ }^{3}$ The Canterbury Initiative, Canterbury District Health Board, Christchurch, New Zealand

${ }^{4}$ Canterbury Clinical Network, Canterbury District Health Board, Christchurch, New Zealand

${ }^{5}$ Pegasus Health Ltd, Christchurch, New Zealand

Correspondence to Dr Michael Epton; michael.epton@cdhb.health. $\mathrm{nz}$

\section{BACKGROUND}

On 22 February 2011, a 6.3 magnitude earthquake hit directly under Christchurch, New Zealand, at a depth of $5 \mathrm{~km}$. The central city was essentially destroyed, with 1240 buildings in the area being immediately destroyed, or subsequently requiring demolition. ${ }^{1}$ In total, 400000 tons of liquefaction silt was squeezed out of the ground, requiring removal and disposal. Christchurch Hospital was partially cases over the next few days. In total, 9000 rooms needed repairs, 106 hospital beds (17\% of total) were closed, 19 community pharmacies and 5 general practices were lost or damaged, and 635 rest home beds were closed. To compound problems, the earthquake caused flooding in the basement of the evacuated, but still handled 220 major trauma

\section{Key messages}

Could exacerbations of COPD be safely managed in non-hospital settings, following the devastating Christchurch Earthquake of 2011?

- A significant reduction in COPD admissions and day stay was able to be achieved by changing systems' responses to COPD exacerbations, following the Christchurch Earthquake of 2011.

- The Christchurch Earthquake of 2011 provided a unique opportunity to alter place of care of COPD exacerbations, leading to significant and sustained reduction in hospital admission and bed-day occupancy, safely undertaken by altering systems responses, rather than specific patient treatment.

hospital, requiring services to pump $80000 \mathrm{~L}$ of water out each day.

Unsurprisingly, given, among other factors, the population's fear of being in large buildings during aftershocks, overall all-cause admission rates dropped dramatically immediately after the earthquake, settling at $\sim 13 \%$ lower than prior to the event. The only exception to this trend was admissions for chronic obstructive pulmonary disease (COPD), which remained at exactly the same level as prior to the earthquake, with no change in overall bed-day occupancy for COPD admissions. At the time of the earthquake, each year, about 800 individuals would be admitted to Christchurch Hospital (catchment area approximately 500000 people) with exacerbations of COPD, for an overall total number of admissions of $\sim 1250$ per year. Average day stay for COPD at the time of the earthquake was 4.5 days, leading to a total bed-day occupancy for COPD of $\sim 5600$ bed-days per year. The Health Quality and Safety Commission New Zealand has previously identified COPD admissions as the most amenable to community change to prevent admission (https://www.hqsc.govt. nz/our-programmes/health-quality-evaluation/projects/atlas-of-healthcare-variation/ adult-ambulatory-sensitive-hospitalisations/). 
5.1.2 COPD hospital admission rates, population aged 15 and over, 2009 (or nearest year)
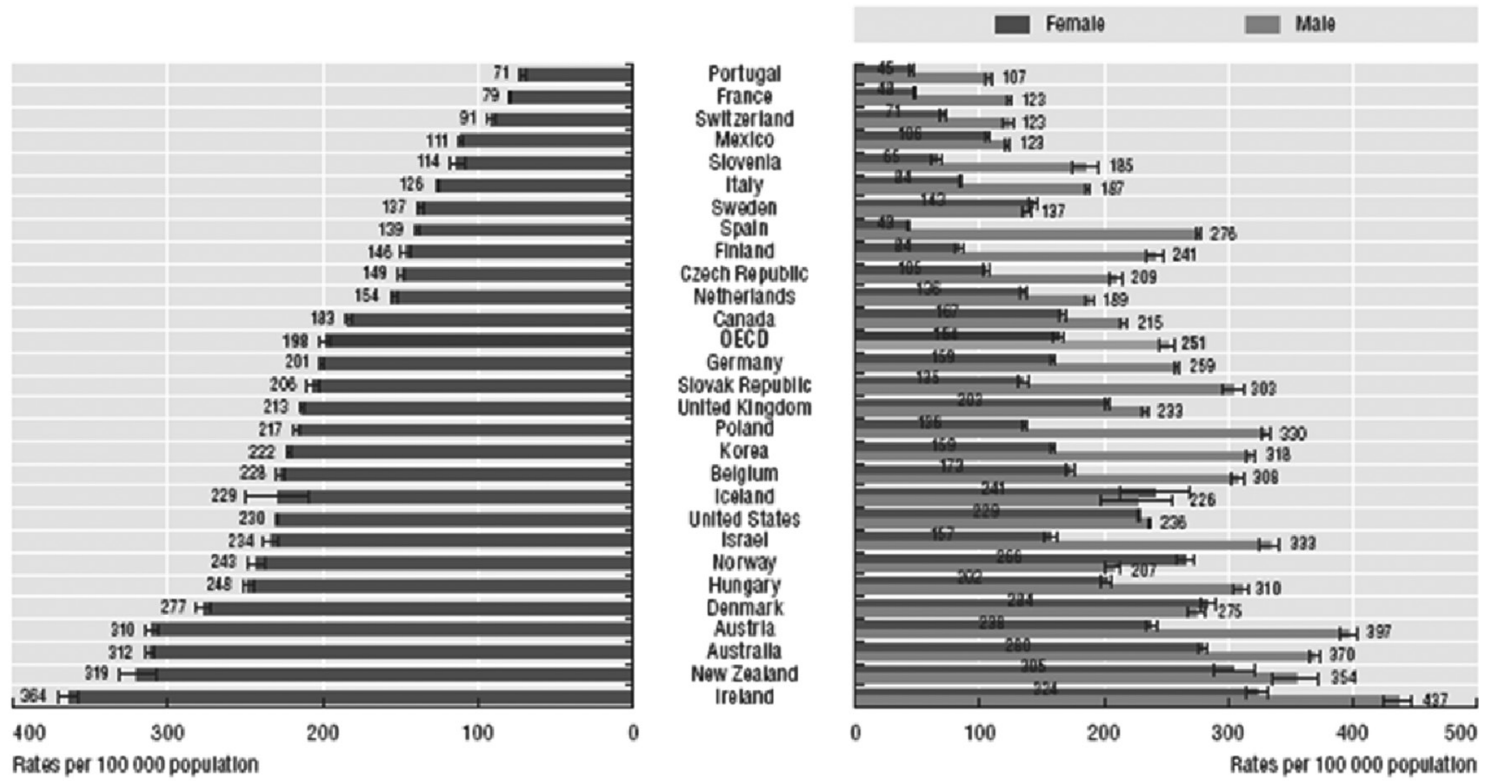

Note: Rates are age-sex standardised to 2005 OECD population. $95 \%$ confidence intervals are represented by 1 .

Source: OECD Health Data 2011.

Figure 1 Chronic obstructive pulmonary disease (COPD) hospital admission rates, population aged 15 and over, in Organisation for Economic Co-operation and Development (OECD) countries in 2009 (or nearest year).

\section{The immediate postearthquake situation}

Given the need to conserve the remaining hospital beds for patients with highest acuity medical and surgical conditions, attention focused on COPD admissions, in an attempt to understand the issue, and why bed-day occupancy had not changed. A taskforce including clinicians, health administrators and funders from all sectors of the health system was formed in the aftermath of the earthquake to better understand the factors leading to admission to hospital with COPD and to attempt to safely reduce admission and bed occupancy for COPD at Christchurch Hospital. Local data to inform these discussions came from a combined database of hospital admissions, emergency department usage and ambulance callouts. This combined database was explored using a linked database analysis tool (Lightfoot Solutions, Bracknell, UK; http:/ /www.lightfootsolutions.com/).

On review of international admissions data (figure 1), it became clear that New Zealand hospitals had some of the highest admission rates for COPD per head of population in the Organisation for Economic Co-operation and Development. ${ }^{2}$ Although there is a greater than four-fold variance in COPD admission rates between countries, this is not explained by smoking rates or COPD prevalence. For example, hospitals in Portugal admit 71/100 000 people compared with 319/100 000 people in New Zealand. In addition, it had previously been recognised that, in Christchurch, admissions with mild exacerbations of COPD were increasing, whereas severe exacerbations were stable, in spite of increasing prevalence and severity of the underlying disease in the population (figure 2). ${ }^{3}$
Further study of transport options to hospital showed that $>80 \%$ of all presentations to the emergency department (ED) for COPD in Christchurch were as a result of an ambulance call. Most ambulances were called by patients themselves using the 111 emergency number, as opposed to being called by their general practitioner (ie, patients were using the ambulance service as their first point of contact during an exacerbation). There was also a difference in admission frequency depending on the type of first response by the ambulance service. Attendance by a 'first responder' SUV-type ambulance (often staffed by more experienced ambulance officers) was

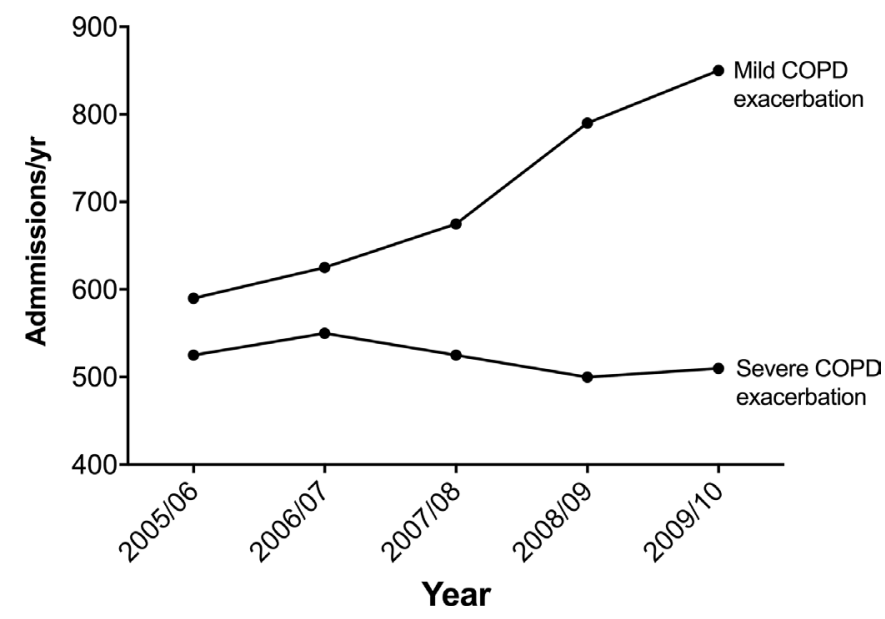

Figure 2 Admissions for mild and severe exacerbations of chronic obstructive pulmonary disease (COPD) in Canterbury between 2005 and 2010 using the AR-DRG criteria E65A for severe COPD and E65B for mild COPD. 


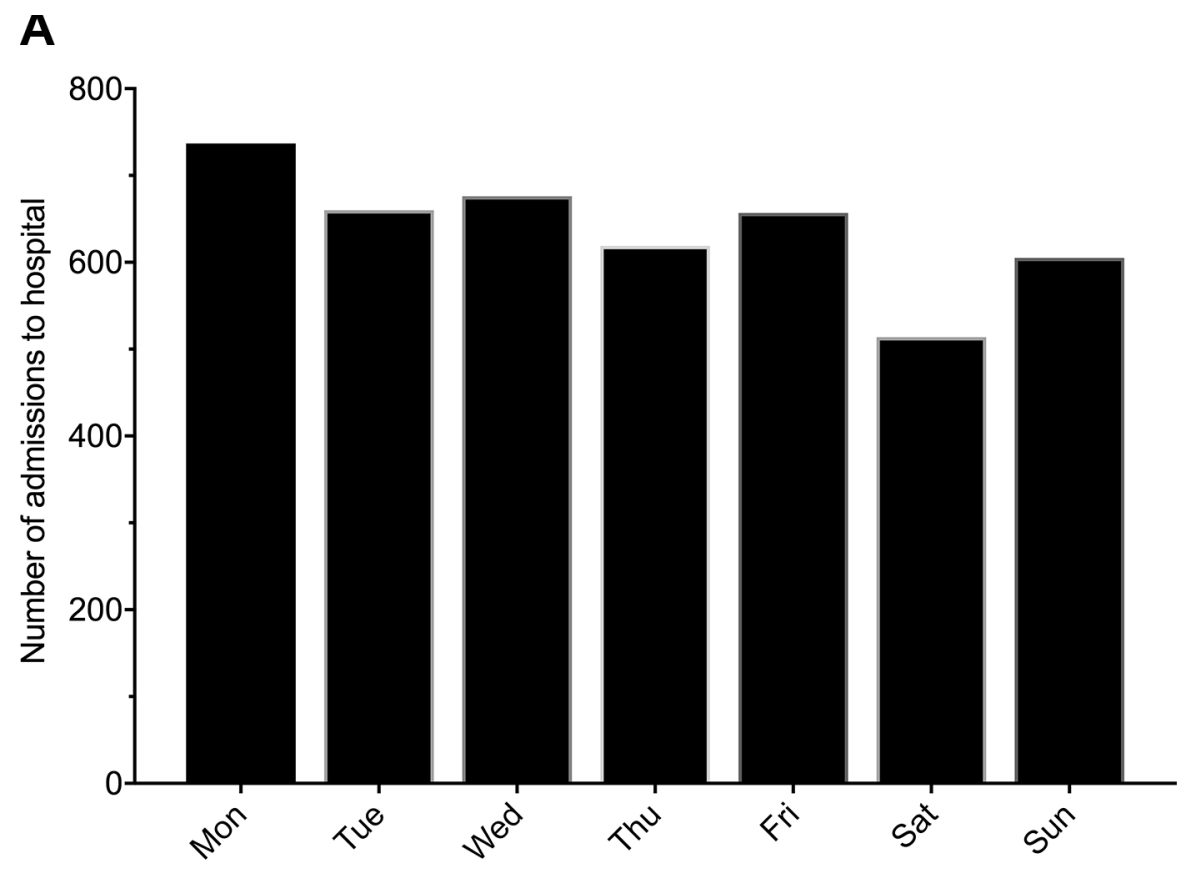

B

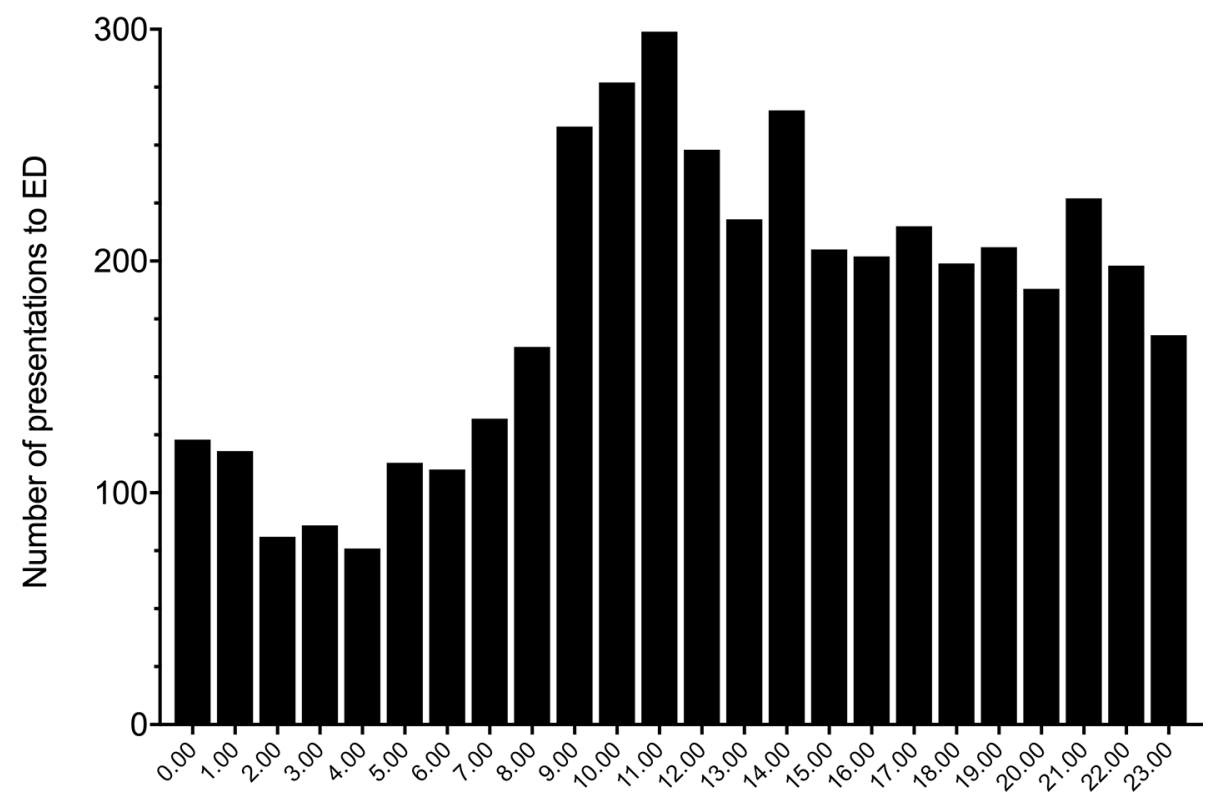

Figure 3 Patients with exacerbations of chronic obstructive pulmonary disease admitted to hospital according to the day of the week (A), and presenting to the emergency department (ED) according to the time of day (B) for the 4-year period, July 2008-July 2012.

associated with lower rates of transport to the ED than if the first response was by a standard ambulance with transportation capacity.

We hypothesised that 111 calls and ambulance attendance occurred because patients were seeking help out-of-hours when their doctor was not available. This proved not to be the case, with Saturday and Sunday being the quietest days for ED admissions, and Monday being the busiest (figure $3 \mathrm{~A}$ ). The busiest time of the day for presentation at the ED with an exacerbation of COPD was between 09:00 and midday, rather than at night, as previously assumed (figure 3B).

Once in the ED with COPD, admission was almost certain, with $85 \%$ of ED presentations subsequently being admitted. This compares with $50 \%$ of presentations to the ED with asthma being admitted. One proposed reason for this was the New Zealand Ministry of Health requirement that a decision about admission versus discharge 
from ED be made within 6 hours. ED staff were reluctant to discharge patients with COPD with ongoing symptoms, hence high rates of admissions, even for physiologically mild exacerbations of COPD.

Once in hospital, there are considerable barriers to discharge, not least inadequate availability of community services over weekends. This leads to patients staying on wards over the weekend, so that a major driver for day stay in COPD is the day of the week a patient is admitted. ${ }^{4}$

As a consequence of this initial analysis, we realised that all responses of the healthcare system in New Zealand, for exacerbations of COPD, were uninitentionally skewed to make admission the default option for care, irrespective of the severity of the exacerbation and the availability of community care options. We recognised that lower admission rates reported in other countries were likely due to different approaches of their healthcare system that made admission less likely. The fact that most attendances and admissions occurred during office hours was encouraging since this meant we would not need to develop a stand-alone response to COPD after hours (ie, a COPD 'flying squad'), but could alter responses to allow patients to be managed in community settings using pre-existing services. We hypothesised that patients could be managed safely in the community, mostly because other patients with similar severity of COPD exacerbations were already being managed in primary care, both in New Zealand (those people who did not use the 111 service as their first point of contact) and overseas (because of lower overall admission rates).

\section{Development of the new service model}

A taskforce was established to explore new service delivery models, culminating in a meeting, attended by a broad range of stakeholders, including ambulance services, primary and secondary care, emergency department, community acute care providers, managers and funders, and, most importantly, patients with COPD. This determined a new model of care which was to be adopted in early winter 2012. This model proposed a number of changes across the patient journey for an exacerbation of COPD, with agreed responses and communication pathways. These responses included enhanced ambulance triage and alternative response pathways, increased use of community acute care services (Acute Demand) and increased transport to the patient's general practice in the event of a mild exacerbation. In addition, we generated patient-specific information sources, both held by the patient, and on the electronic medical record, with knowledge of the patient's baseline status guiding triage.

\section{Ambulance triage and alternate responses}

An agreed triage tool was developed with ambulance services, based on the Modified Early Warning score system (figure 4). ${ }^{5}$ This triage tool was laminated and carried in all ambulances in Christchurch. This allowed ambulance officers to classify the severity of the exacerbation as mild, moderate or severe, and gave options for where the patient might be managed, including stay at home with support, transport to the patient's general practitioner (GP), transport to the community-GP mannedemergency facilities, or transport to the ED. It was agreed that the triage criteria should be conservative, given the skillset of an attending ambulance officer, and the potential difficulties recognising severe exacerbations of COPD. It was made clear that this triage process was only for patients with clearly diagnosed COPD and was not to be used for other conditions such as asthma. In addition to the triage template, communication pathways were defined between ambulance services and community acute care facilities and specialist services, to allow discussion of uncertainty in triage.

\section{The COPD Blue Card}

One issue that was highlighted as part of initial discussions was that ambulance services and other first responders frequently did not know the patient's baseline physiological status, especially baseline oxygen saturation. Previous local ambulance protocols had led to the potential for overuse of oxygen which then increased the likelihood of admission. ${ }^{6}$ We hypothesised that, if an ambulance officer recognised that current oxygen saturation was little changed from the patient's baseline (as opposed to 'normal'), this would reduce the overall concern about the severity of the current exacerbation, and hence alter decisions about the necessity for emergency department care. We therefore created a COPD Blue Card (figure 5) in the form of an A5-sized fridge magnet. This gave simple self-management advice on one side, and baseline information about the patient on the other, including physiological information, and the patient's management wishes. This Blue Card was distributed to all patients who had been admitted to hospital with COPD in the previous three years, and patients were encouraged to meet with their GP to discuss and fill in the form. This patient group was targeted as the group most likely to require ambulance services in the future. Blue Cards were also distributed to general practices, to use and fill in at their discretion, for patients identified as being potentially at risk of admission.

\section{Acute demand and community nurses in hospital settings}

We recognised that ED staff were using admission as a default option for COPD exacerbations due to a number of factors, specifically the perceived difficulty accessing community acute care services by comparison with admission. For this reason, we stationed nurses trained in community care within the ED to work with other staff to access community care for patients who had presented with a mild exacerbation. Having community-trained nurses and GPs in acute care settings had already been shown in other diseases such as cellulitis ${ }^{7}$ and pneumonia, ${ }^{8}$ to increase acceptance of community management options. The acute demand service had already 


\begin{tabular}{|c|c|c|c|}
\hline & Mild & Moderate (ANY OF) & Emergency (ANY OF) \\
\hline GCS & & & $\begin{array}{l}<14 \text { Drowsy/Confused/ } \\
\text { Comatose }\end{array}$ \\
\hline Talking & Sentences or phrases & Phrases & Words or Respiratory Arrest \\
\hline Temperature & Afebrile & $\begin{array}{l}\text { Afebrile or low grade fever } \\
(<38)\end{array}$ & Febrile $(>38)$ \\
\hline Respiratory Rate & $<20$ & $21-30$ & $>30$ or Respiratory Arrest \\
\hline Oxygen saturations & $\begin{array}{l}\text { Within } 5 \% \text { of known } 02 \text { sats } \\
\text { when stable } \\
\text { AND } \\
\text { Above } 88 \%\end{array}$ & $\begin{array}{l}5 \% \text { below known stable } \\
\text { 02sats } \\
\text { OR } \\
\text { Below } 88 \%\end{array}$ & \\
\hline Other & $\begin{array}{l}\text { Examination consistent } \\
\text { with COPD, with no other } \\
\text { concerning features }\end{array}$ & $\begin{array}{l}\text { Any feature not consistent } \\
\text { with COPD }\end{array}$ & $\begin{array}{l}\text { Hypotensive/Shocked/BP } \\
<100 \text { systolic }\end{array}$ \\
\hline $\begin{array}{l}\text { Pathway } \\
\text { recommendation }\end{array}$ & $\begin{array}{l}\text { - } \text { - Lim to manage at home } \\
\text { instance } \\
\text { Referral to ADMS for RN } \\
\text { visit within } 4 \text { hours } \\
\text { Patient advise: If unwell } \\
\text { before } R N \text { visit to call } 111\end{array}$ & - Transport to $24 \mathrm{hr}$ Surgery & $\begin{array}{l}\text { - Transport to } \mathrm{CHCH} \text { ED } \\
\text { Canterbury Network } \\
\text { v1 May } 2012\end{array}$ \\
\hline
\end{tabular}

Figure 4 Image of the form used by ambulance officers to triage patients with an exacerbation of chronic obstructive pulmonary disease (COPD). GP, general practitioner; GCS, Glasgow Coma Scale; ADMS, Acute Demand Medical Service; $\mathrm{RN}$, Registered Nurse; $\mathrm{CHCH}$, Christchurch
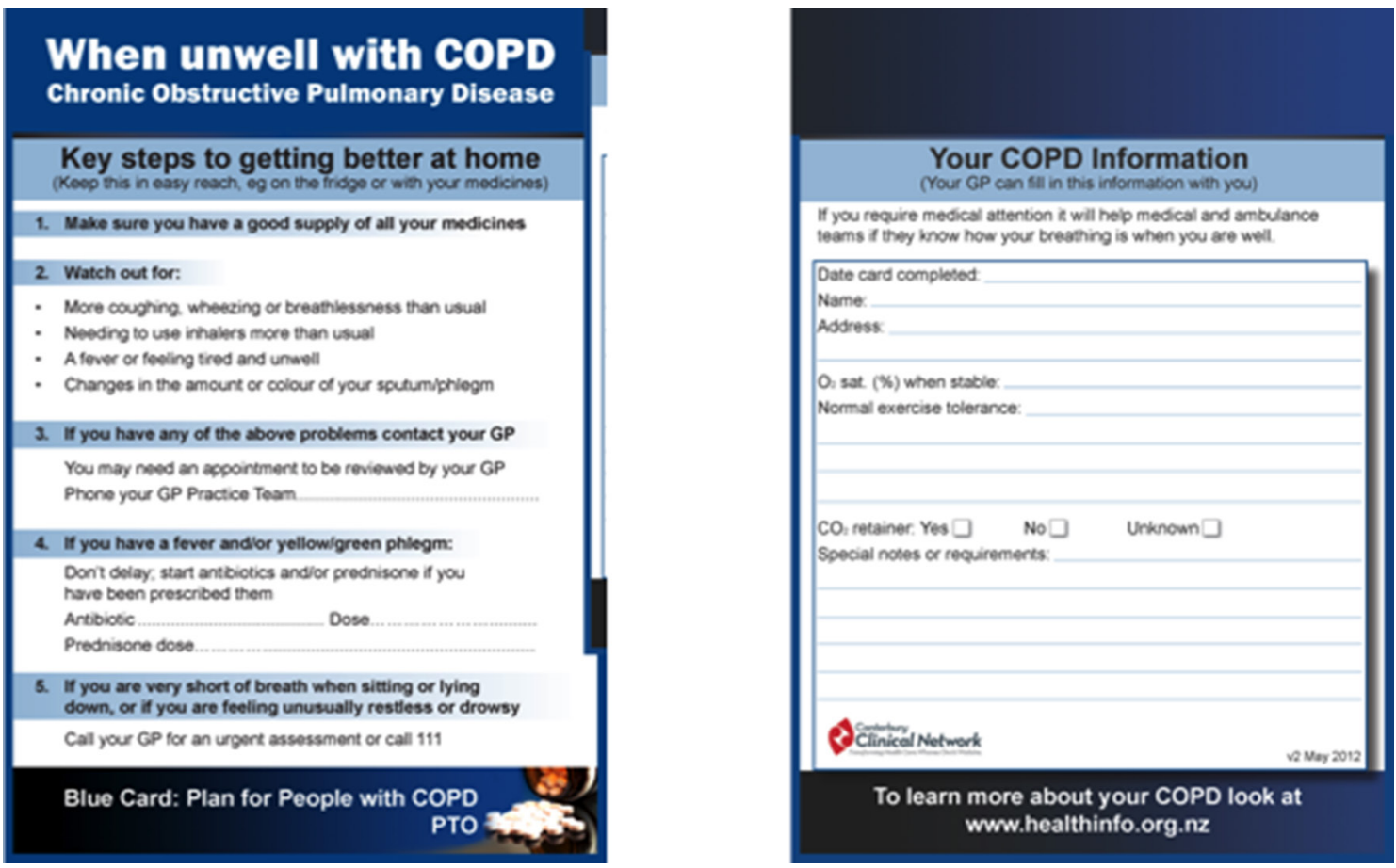

Figure 5 Image of the Blue Card provided to patients with chronic obstructive pulmonary disease (COPD). 
increased capacity and funding to manage patients with other conditions in the community, following the earthquake. ${ }^{9}$ These nurses were directed to pay specific attention to exacerbations of COPD, exploring community care options with the patient and staff in the ED, and to link with the acute demand service.

\section{Communication pathways}

Underlying all of these changes were clear pathways for communication and support, both for individual patient care decisions, but also for operational and safety concerns. These pathways emphasised early phone contact, at all stages through the patient journey. 'Who do you call if you have concerns?'. One issue recognised by the initial stakeholder discussions was a decision that had been made by ambulance services not to use cell phones for communication, but only use two-way radio to the ED and ambulance base. This decision had been made because of difficulty deciding how to pay for calls made by ambulance officers using their own personal cell phones. To remove this barrier, the taskforce agreed that any call made on a personal cell phone for patient-related purposes would be reimbursed. At the time of writing, no reimbursement has been required, indicating that this barrier was perceived rather than real.

\section{OUTCOMES}

\section{COPD admissions}

The new COPD care pathway went live in end July 2012 (Southern hemisphere winter). Table 1 shows the initial destination of patients in the 3.5 months after this point.

As can be seen, community management of COPD exacerbations which had resulted from a 111 call increased from $19 \%$ to $42 \%$. Of interest, the majority of care of these exacerbations occurred in general practice, that is, the exacerbations were classified as mild by the ambulance officers. This allowed care of mild COPD to be managed in multiple general practices, rather than the ED or community acute care service settings, which are likely to be overwhelmed, especially during winter.

The effect on hospital admissions for COPD is shown in figure 6. An initial peak, associated with an influenza outbreak in August 2012, led to higher admission numbers than predicted, followed by a marked reduction in the number of admissions below that expected. Over the subsequent 5 years, admissions have remained markedly lower than predicted, with a major impact on bed-day occupancy for COPD and overall bed-day occupancy decreasing from a peak of $\sim 8200$ bed-days per year in 2012 to 4230 in 2016 (figure 7). This reduction in bed-days, at approximately NZ $\$ 1500$ per day, ${ }^{10}$ freed up NZ\$6 million per year from budgets to be allocated elsewhere, in addition to freeing up beds for other patients.

Initially there were concerns from general practices about the increased workload that this initiative might involve. These concerns were allayed by pointing out that as a consequence of ambulance diversion each individual GP might be expected to see one extra patient with an exacerbation of COPD per year. In addition, it was accepted that the severity of the exacerbations being transferred to the care of the patient's GP would be mild, similar to the severity of exacerbations already being seen as routine practice. Subsequent discussions when the initiative had been started showed that there was clear support and enthusiasm from general practice as a consequence of these changes. This was because many of the patients previously admitted had little or no previous regular contact with their GP. This ambulance diversion in an exacerbation brought patients into contact with their GP and practice, allowing other interventions to occur (smoking cessation, influenza vaccination and social supports), and improving future care of that patient.

\section{Safety}

Due to the disruption to services after the earthquake, we did not have the resource to undertake a full safety and monitoring audit for each patient managed as part of this pathway change. We did however undertake assessment of any reported serious adverse outcomes, including one death. In this latter case, the process of management including triage status was assessed as being appropriate, and in keeping with the patient's wishes for care and transportation. We also captured data about patients who subsequently required transport to hospital, after community treatment, to identify potential treatment 'failure' in community settings. This onward transport was usually low at $8 \%-10 \%$ of overall ambulance activity.

In addition, we were not able to undertake a formal quality assurance process assessing patient and healthcare worker satisfaction with the new service, but relied

Table 1 Location of initial treatment of chronic obstructive pulmonary disease exacerbations before and after introduction of the programme

\begin{tabular}{lcccccc}
\hline & Kept at home & General practitioner & 24hours surgery & Emergency department & Total & \% treated in community \\
\hline July & 2 & 4 & 38 & 186 & 230 & 19 \\
August & 7 & 62 & 16 & 116 & 201 & 42 \\
September & 11 & 60 & 14 & 128 & 213 & 40 \\
October & 8 & 57 & 18 & 114 & 197 & 42 \\
November & 4 & 34 & 5 & 55 & 98 & 44 \\
\hline
\end{tabular}




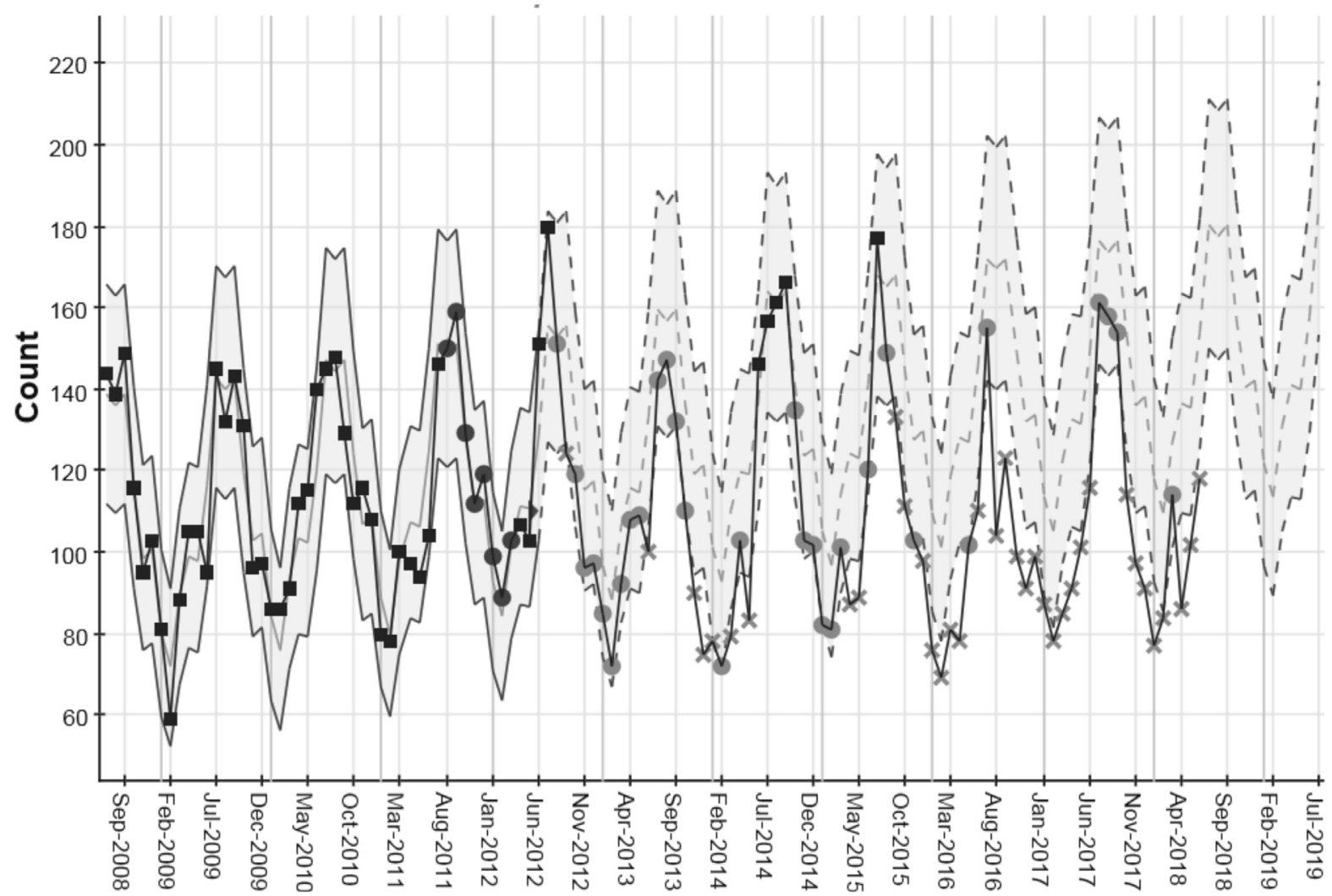

Figure 6 Monthly admissions for chronic obstructive pulmonary disease (COPD) to Christchurch Hospital. Individual data points are charted along with variance from a prediction model. The prediction model for COPD admissions uses data from July 2008 to July 2012 (moving average \pm 3 SD prior to July 2012; dotted lines show predicted admissions \pm 3 SD for COPD from July 2012 using trend data from four previous years). Data points change from dark grey to light grey if $>7$ consecutive data points are below the moving average. If a data point is $>3 \mathrm{SD}$ above or below the moving average, this is expressed as a cross symbol (light grey for below moving average). This allows visualisation of trends towards improvement or worsening of an outcome, and significant outliers.

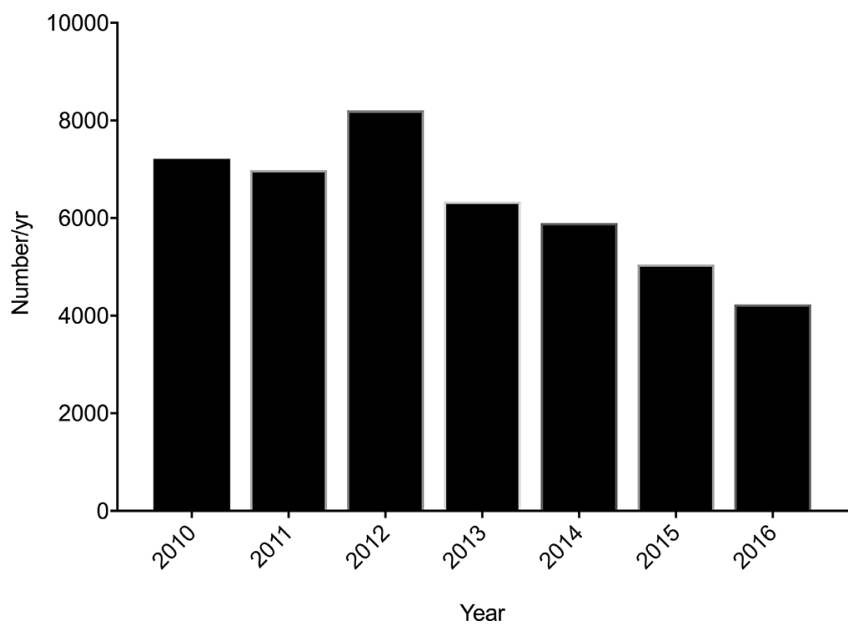

Figure 7 Changes in the number of occupied beddays for chronic obstructive pulmonary disease between 2010 and 2016. on anecdote and personal communications to identify compliments and concerns.

\section{Learnings}

There were a large number of factors that contributed to the success of this initiative, both in the short term, but also sustained positive outcomes. It was very clear that the success related not to alteration in medical management of exacerbations of COPD, but alterations in the systems, processes and attitudes of health professionals across the sector, and patients themselves.

\section{Effective use of admissions and ambulance data}

In Canterbury we have established a linked database analysis tool (Lightfoot Solutions, Bracknell, UK http://www. lightfootsolutions.com/) which enabled detailed analysis and visualisation of all aspects of the 'patient journey' during an exacerbation. This dataset could be queried 
quickly by senior clinicians and managers from the taskforce, enabling challenging or confirming of assumptions about admission behaviours, and a rapid ability to test hypotheses about care. This analysis process occurred very early in the project and allowed senior clinicians in the taskforce to challenge their own assumptions, and also to create a narrative to enable further discussions with stakeholders, allowing rapid behaviour change. Examples of the data visualisation which occurred early in this project can be seen in table 1 and figures 6 and 7 .

\section{Effective sharing of patient data}

While a goal of all healthcare systems is a shared view of an agreed electronic medical record for each patient, this was not available at the time of the Christchurch Earthquake. However, the agreed use of low-tech snapshots of the medical record, that is, the Blue Card, allowed important information about COPD severity to be agreed between the patient and their GP for subsequent use in all acute interactions with the healthcare system. The rollout of Blue Cards was widespread and rapid, and the use of a simple card, as a fridge magnet, allowed easy access to information, encouraging accurate triage and agreed responses by healthcare workers.

\section{Communication}

At all points in the 'patient journey' we established clear communication lines between health professionals looking after the patient, using the mantra 'Who do you call if you are uncertain?' This enabled ambulance officers to contact the patient's GP, or the after-hours service, with confidence, and allowed community care providers to quickly link to specialist advice as required. The overall number of calls made were actually not large, but having the confidence to know someone more experienced was available to guide decisions reduced barriers to effective community care. In addition, we attempted to make communication, as much as possible, face-to-face. This was particularly important between health providers employed by different organisations and allowed ambulance officers to interact directly with hospital clinicians and GPs to air their views and concerns.

\section{Champions}

A critical factor in encouraging these changes was agreed messages championed by a senior respected respiratory physician (ME). These messages were repeated in multiple education events and meetings both in the hospital and in the community. These were reinforced by clinical leaders from general practice, ambulance, ED and general physicians. The key messages included: 'It is not about the physiology but the sociology', 'Call someone if you are uncertain', 'I don't care who you are employed by, I care about what's best for the patient' and 'Patients do not want to be in hospital, they want to be at home'.

\section{Trust}

Culture change was reinforced by frequent debate and updates, including 'real-time' admission data for all stakeholders. We could demonstrate almost immediate and sustained change using graphs similar to those shown in this publication. This visualisation of data reinforced the culture change and supported other stakeholders in the system to trust other providers, even though until that point there had been little interaction between them, for example, between hospital clinicians and the ambulance service. The ongoing communication, supported by data, allowed trusting relationships to develop across all sectors.

\section{The confidence to "let go"}

Many of the implicit assumptions being made throughout the healthcare sector about exacerbations had led to the culture of hospitalisation for exacerbations; this was previously seen to be right and safe and, until the earthquake, was not challenged. For hospital clinicians to see that the data were being understood accurately, and being part of the process of generating agreed community assessment and care pathways, allowed relationship between sectors to evolve, and gave hospital clinicians reassurance that patient care was not being compromised by the new approach.

\section{Myths}

Previous behaviour of the system had been driven by the perception that the increase in admissions had been due to the demographics and increasing severity of the underlying disease in this patient population. The analysis of exacerbation severity as part of the initiative planning process and the subsequent high frequency of mild exacerbations diverted to general practice care showed that, in Christchurch at least, this was a myth, and that, although the severity of the underlying disease might be worsening in the population, the increase in admissions was due to more mild exacerbations being treated in hospital.

In addition, there had been a clear perception that most patients with COPD exacerbations sought ED and hospital help after-hours and on weekends because of unavailability of GP care. This was clearly shown to be false. This allowed care structures to be changed without significant investment in after-hours care. The overall cost of the initiative was therefore low and could be absorbed in current care structures in Christchurch, such as general practice, and Acute Demand services.

\section{CONCLUSIONS}

The Christchurch Earthquake of 2011 provided a unique opportunity to review and alter care of COPD exacerbations, leading to significant and sustained reduction in hospital admission and bed-day occupancy. This was undertaken by altering systems responses, rather than 
patient treatment. Many of the learnings can potentially be transferred to other places, to facilitate discussions about place of care for exacerbations of COPD, and to potentially reduced hospital admissions. We would encourage these discussions and changes to occur without the stimulus of an earthquake in your healthcare environment!

Acknowledgements The authors thank Trevor Read from Lightfoot Solutions New Zealand for his support for rapid and comprehensive data analysis and visualisation using the Lightfoot programme, in the early stages of the Taskforce establishment.

Contributors The work group for programme development consisted of ME as a Community Respiratory Physician in the Canterbury Clinical Network, CL as a Service Improvement Lead for the Canterbury DHB, GM as Clinical Leader in the Canterbury Initiative, RL as GP Liaison for Respiratory and General Medicine in the Canterbury Initiative, SB as Clinical Director of the 24-hr Observation Unit, Pegasus Health, AM as Medical Director of the Acute Demand Management Programme and RN-H the Lead from St John Ambulance, Christchurch. ME and BS collected and generated the data and wrote the article. All authors contributed to the development of the manuscript and approved the final version.

Funding The study was funded by the Canterbury Distict Health Board.

Competing interests None declared.

Patient consent Not required.

Provenance and peer review Not commissioned; externally peer reviewed.

Data sharing statement No additional data are available.

Open access This is an open access article distributed in accordance with the Creative Commons Attribution Non Commercial (CC BY-NC 4.0) license, which permits others to distribute, remix, adapt, build upon this work non-commercially, and license their derivative works on different terms, provided the original work is properly cited, appropriate credit is given, any changes made indicated, and the use is non-commercial. See: http://creativecommons.org/licenses/by-nc/4.0/

\section{REFERENCES}

1. McIntosh JK, Jacques C, Mitrani-Reiser J, et al. The impact of the 22nd February 2011 Earthquake on Christchurch Hospital. University of Canterbury Research Repository. 2012 https://ir.canterbury.ac.nz/ handle/10092/7284

2. OECD. Health at a Glance 2011: OECD Indicators, OECD Publishing $2011 \mathrm{http}: / / \mathrm{dx}$.doi.org/10.1787/health glance-2011-en

3. New Zealand Ministry of Health. AR-DRG v4.2 codes. 2017 http:// www.health.govt.nz/nz-health-statistics/data-references/diagnosisrelated-groups/australian-refined-diagnosis-related-groups-v70-ardrg-v70

4. Roberts CM, Lowe D, Skipper E, et al. Effect of time and day of admission on hospital care quality for patients with chronic obstructive pulmonary disease exacerbation in England and Wales: single cohort study. BMJ Open 2017;7:e015532.

5. Subbe CP, Kruger M, Rutherford P, et al. Validation of a modified early warning score in medical admissions. QJM 2001;94:521-6.

6. Wijesinghe M, Perrin K, Healy B, et al. Pre-hospital oxygen therapy in acute exacerbations of chronic obstructive pulmonary disease. Intern Med J 2011;41:618-22.

7. Corwin P, Toop L, McGeoch G, et al. Randomised controlled trial of intravenous antibiotic treatment for cellulitis at home compared with hospital. BMJ 2005;330:129.

8. Richards DA, Toop LJ, Epton MJ, et al. Home management of mild to moderately severe community-acquired pneumonia: a randomised controlled trial. Med J Aust 2005;183:235-8.

9. Gullery C, Hamilton G. Towards integrated person-centred healthcare - the Canterbury journey. Future Hospital Journal 2015;2:111-6.

10. World Health Organisation. Choosing intervention that are cost effective (WHO-CHOICE), 2017. http://www.who.int/choice/country/ $\mathrm{nzl} /$ cost/en/ 